\title{
The Role of Angiopoietin-like protein 3 and Fibroblast Growth Factor 21 to Lipolysis, Inflammation and Insulin Resistance in Indonesian Non-Diabetic Obese Male
}

\author{
Yani Lina ${ }^{1,2 *}$, Gatot S. Lawrence ${ }^{1}$, Andi Wijaya ${ }^{1,2}$, and Suryani As'ad ${ }^{1}$ \\ 'Post Graduate Program in Biomedical Science-Clinical Chemistry, Faculty of Medicine, Hasanuddin University, Makassar \\ ${ }^{2}$ Prodia Clinical Laboratory, Jakarta \\ *Address correspondence to this author at: Prodia Clinical Laboratory, JI. Kramat Raya 150, Jakarta, Indonesia \\ E-mail: yani_lina1979@yahoo.com
}

\section{Abstract}

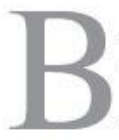

ACKGROUND: Obesity is commonly associated with a systemic low grade inflammation and insulin resistance state. Although it is still being debated, increased lipolysis is known as one of the risk factors for inflammation and insulin resistance. Two factors already known to affect lipolysis are Angpt13, known as prolipolytic factor, and FGF21, known as antilipolytic factor. The aim of this study was to observe the role of Angpt13 and FGF21 to lipolysis, inflammation and insulin resistance in non diabetic obese male.

METHODS: This was an observational study with cross sectional design. One hundred and thirty male subjects aged $30-60$ years with non diabetic abdominal obesity characterized by waist circumference $97.32 \pm 5.63 \mathrm{~cm}$ and fasting blood glucose $90.19 \pm 8.78 \mathrm{mg} / \mathrm{dL}$.

RESULTS: The results of this study showed a correlation between Angpt13-FFA ( $\mathrm{r}=0,203 ; \mathrm{p}=0,021 ; R$ square $0,041 ; \mathrm{p}=0,021)$, Angptl3-FABP4 ( $\mathrm{r}=0,330 ; \mathrm{p}=0,000 ; R$ square 0,$109 ; \mathrm{p}=0,000)$ and Angpt13-TNF $\alpha(\mathrm{r}=0,288 ; \mathrm{p}=$ $0,001 ; R$ square 0,$049 ; \mathrm{p}=0,011)$. There was a correlation between FGF21-FABP4 ( $\mathrm{r}=0.218, \mathrm{p}=0,013 ; R$ square $0,047, \mathrm{p}=0,013)$ and FGF21-HOMA-IR $(\mathrm{r}=0,308, \mathrm{p}=$ $0,000 ; R$ square $0,046, \mathrm{p}=0,014)$.
CONCLUSIONS: We conclude that Angpt13 may affect lipolysis and inflammation while FGF21 may affect lipolysis and insulin resistance. The increased FGF21 concentration might occur as a compensation (negative feedback mechanism) to reduce lipolysis and increase insulin sensitivity in non diabetic obese males. Further studies might be needed to observe Angpt13 and FGF21 profile in more severe obese population in Indonesia.

KEYWORDS: Obesity, lipolysis, inflammation, insulin resistance.

\section{Introduction}

The prevalence of obesity especially abdominal obesity in Indonesia has increased during the past ten years. Greater abdominal adiposity is strongly associated with insulin resistance, dyslipidemia, and systemic low grade inflammation, which play essential roles in the pathogenesis of cardiovascular disease (CVD), metabolic syndrome, and certain cancers (1).

Although it is still being debated on how accumulation of subcutaneous and/or visceral fat produces insulin resistance (which occurs predominantly in skeletal muscle), it has nevertheless become clear that elevated plasma levels of free fatty acids (FFA) are a major link between obesity and insulin resistance/T2DM. Plasma levels of FFA are commonly higher in obese as compared with non-obese people. The reason for this includes an increased number 
of fat cells, a decrease in FFA clearance and at a later stage insulin resistance at the adipose tissue level resulting in increased lipolysis. There is strong evidence that FFA can inhibit various actions of insulin in several target tissues including skeletal muscle, liver, and vascular endothelial cells (2).

The proinflammatory cytokine TNF- $\alpha$ is another important contributor to the development of insulin resistance. TNF- $\alpha$ level is elevated in adipose tissue of various obese rodent models and in obese humans, whereas a genetic defect in TNF $-\alpha$ signaling significantly improves IR signaling capacity and insulin sensitivity in diet induced and genetically obese mice. Nguyen's study indicates that 1) stress $-\alpha$ activated protein or c-Jun amino-terminal kinases (JNK) can be activated by FFA through TNF- $\alpha$ -independent mechanisms, 2) activated JNK is a major contributor to FFA-induced cellular insulin resistance, and 3) TNF- $\alpha$ is an autocrine/paracrine downstream effector of activated JNK that can also mediate insulin resistance (3).

The adipocyte fatty acid binding protein (aP2; also known as FABP4 and ALBP) was first detected in adipose tissue. Expression of FABP4 is highly regulated during differentiation of adipocytes and its messenger RNA is transcriptionally controlled by fatty acids. Expression of FABP4 in adipocytes has been shown to promote lipolysis, increased tumor necrosis factor (TNF)- $\alpha$ expression, and contribute to insulin resistance. Macrophage FABP4 may influence foam cell formation and atherogenesis through the effects on FFA shuttling that cause increased cholesteryl ester (CE) formation and/or effects on gene transcription that promote production of inflammatory cytokines. FABP4 may possibly affect genes that are involved in cholesterol homeostasis (4).

The HOMA model is used to estimate insulin sensitivity and $\beta$-cell function from fasting plasma insulin and glucose concentrations. The equations widely used and simplified are: HOMA-IR = (FPI x FPG) $/ 22.5$ for insulin resistance (IR) where FPI is fasting plasma insulin concentration (mU/L) and FPG is fasting plasma glucose (mmol/L) (5).

Angiopoietin-like proteins (Angptls) are orphan ligands with structural similarity to angiopoietins. Angpt13 is now understood to be a hepatocyte-derived circulating factor that functions to regulate lipid metabolism. A study by Shimamura et al. reveals that Angpt 13 targets on adipose cells directly and enhances the lipolysis leading to increased release of FFA and glycerol from adipose cells (6-7).

The human genome encodes 22 members of the fibroblast growth factor (FGF) family. Most function in diverse processes such as development and wound healing. But three members-FGF19 (FGF15 in the mouse), FGF21, and FGF23 - have recently emerged as metabolic hormones (8). Arner's study showes that FGF21 attenuates hormone-stimulates lipolysis in both human and murine adipocytes. In vivo this triggers reduction of FFA release and can thereby contribute to the insulin sensitizing effect of FGF21. The decrease in lipolysis can also contribute to reduced lipotoxicity on islet cells in addition to the direct effects of FGF21 recently shown in beta cells (9).

\section{Methods}

\section{STUDY DESIGN}

We used observational study with cross sectional design on 130 obese male volunteers. Data collection was carried out from January to June 2009. The study proposal was approved by the Health Research Ethics Committee of the Faculty of Medicine, University of Hasanuddin, Makassar, Indonesia.

\section{SUBJECTS}

The study subjects were recruited from clients coming to Prodia Clinical Laboratory, Jakarta, Indonesia, for general medical check-up, as well as employees and relatives of this Laboratory. The subjects apparently healthy, male, aged 30-60 years, with WC $>90 \mathrm{~cm}$, and willing to follow the study protocol, were considered eligible to be included in the study.

Subjects who were consuming steroids; or had consumed antioxidant supplements such as vitamins $C$ and or $\mathrm{E}$, green tea or other supplements known as antioxidants for the last 3 weeks; or had been treated with antiinflammatory drugs such as statins or thiazolidinedione for the last 3 weeks; or had liver dysfunction, kidney dysfunction, asthma, fever, and other acute inflammation were excluded from the study. Each subject was given explanation about the study and signed an informed consent prior to the commencement of the study.

\section{ANTHROPOMETRIC MEASUREMENT}

Body weight (BW) was measured in kilograms to the nearest $0.1 \mathrm{~kg}$, with light clothes on, using a beam scale Tanita (Tanita, Japan). Height (Ht) was measured in centimeters to the nearest $0.1 \mathrm{~cm}$, in standing position with socks and shoes removed, using a microtois (stature meter). Waist circumference (WC) was measured in centimeters to the nearest $0.1 \mathrm{~cm}$, using a flexible non-elastic tape made 
by Roche (Roche, Switzerland). WC was measured at the midway region between the lowest rib margin and the iliac crest, in standing position with abdomen relaxed, feet close together and weight equally divided over both legs. BMI was calculated by dividing body weight (in $\mathrm{kg}$ ) by height (in squared meter).

\section{BLOOD PRESSURE MEASUREMENT}

Blood pressure was measured using a sphygnomanometer during the course of the study. Subjects were seated for at least $5 \mathrm{~min}$ before the actual measurement was taken. The first Korotkoff sound represented systolic blood pressure and the fifth Korotkoff sound (when sound just disappears) was taken as the diastolic blood pressure. Two measurements were taken with a $5 \mathrm{~min}$ interval, and the average value was used.

\section{BIOCHEMICAL ASSESSMENT}

Fasting blood samples were collected in the morning between 07:00 and 10:00 am. Plasma and serum were separated immediately by centrifugation and aliquots were frozen at $-200 \mathrm{C}$ for subsequent batched analysis for Angpt13, FGF21, FFA, FABP4, and TNF- $\alpha$. Fasting insulin, fasting glucose, hsCRP, creatinine, alanine amino transferase (ALT) and aspartate amino transferase (AST) levels were assayed by the Prodia Clinical Laboratory's routine chemistry procedures. Serum AST, ALT and creatinine were determined using the International Federation of Clinical Chemistry (IFCC) method and reagents manufactured by Roche Diagnostic GmbH. The standard automated glucose oxidase method was used to determine glucose, reagents manufactured by Roche Diagnostic $\mathrm{GmbH}$. The concentration of CRP was determined using a high-sensitivity chemiluminescent assay on the Immulite ${ }^{\circledR}$ (Dade-Behring, Los Angeles, CA, USA). Fasting insulin was measured using ultrasensitive insulin ELISA from Mercodia. FGF21, FABP4 and Angpt13 were measured using an enzyme-linked immunoassay (ELISA) kit from Biovendor. FFA was measured using optimized enzymatic colorimetric assay from Roche Diagnostic GmbH. TNF- $\alpha$

\section{Table 1. Basic Clinical and Biochemical Variables}

\begin{tabular}{lrrrrrr}
\hline Variable & $\mathbf{n}$ & Mean & SD & Median & Minimum & Maximum \\
\hline Age $($ years $)$ & 130 & 38.64 & 5.74 & 38 & 30 & 56 \\
SBP $(\mathrm{mmHg})$ & 130 & 115.12 & 13.53 & 110 & 90 & 150 \\
DBP $(\mathrm{mmHg})$ & 130 & 76.31 & 9.89 & 80 & 60 & 110 \\
Height $(\mathrm{cm})$ & 130 & 166.39 & 5.18 & 166 & 156 & 185 \\
Weight $(\mathrm{kg})$ & 130 & 79.90 & 7.98 & 78 & 65 & 120 \\
WC $(\mathrm{cm})$ & 130 & 97.32 & 5.63 & 96 & 91 & 126 \\
hsCRP $(\mathrm{mg} / \mathrm{L})$ & 130 & 2.19 & 1.83 & 1.55 & 0.22 & 9.12 \\
Bilirubin $(\mathrm{mg} / \mathrm{dL})$ & 130 & 0.23 & 0.07 & 0.22 & 0.09 & 0.46 \\
AST $(\mathrm{U} / \mathrm{L})$ & 130 & 28.92 & 8.09 & 27.50 & 16 & 60 \\
ALT $(\mathrm{U} / \mathrm{L})$ & 130 & 37.58 & 14.32 & 36.50 & 14 & 84 \\
Glucose $(\mathrm{mg} / \mathrm{dL})$ & 130 & 90.19 & 8.78 & 89 & 74 & 119 \\
FFA $(\mathrm{mM})$ & 130 & 0.58 & 0.19 & 0.58 & 0.18 & 1.12 \\
FABP4 $(\mathrm{ng} / \mathrm{mL})$ & 130 & 25.31 & 7.34 & 24.90 & 11.38 & 49.53 \\
Angptl3 $(\mathrm{ng} / \mathrm{mL})$ & 130 & 321.99 & 89.21 & 310.05 & 139.10 & 600.60 \\
FGF21 $(\mathrm{pg} / \mathrm{mL})$ & 130 & 217.93 & 125.36 & 204.31 & 7.00 & 561.34 \\
TNF- $\mathrm{p}(\mathrm{pg} / \mathrm{mL})$ & 130 & 2.80 & 1.05 & 2.47 & 1.33 & 6.50 \\
HOMA-IR & 130 & 2.10 & 1.52 & 1.71 & 0.52 & 8.89 \\
GFR & 130 & 106.22 & 22.35 & 102.05 & 66.07 & 239.35 \\
\hline
\end{tabular}

SBP = Systolic Blood Pressure; DBP = Diastolic Blood Pressure; WC = Waist Circumference; GFR = Glomerular Filtration Rate. 
was measured using sandwich enzyme immunoassay from R\&D Systems Inc. All biochemical analyses were performed at Prodia Clinical Laboratory located in Jakarta, Indonesia.

\section{DATA ANALYSIS}

Data analysis was done using SPSS 11.5 statistical analysis software for Windows (SPSS Inc., Chicago, IL, USA). Distributions of continuous variables were assessed for normality using the Kolmogorov-Smirnov. Associations between variables were analyzed using Pearson and Spearman's correlation analysis. All tests were two-sided and considered significant at $p<0.05$. We also used regression analysis between variables.

\section{Results}

Basic clinical and biochemical variables are shown in Table 1. In this study, we found that most of the parameters used had median below mean concentration especially age and waist circumference so we suggested that our population was still at early and mild obesity stage.

Table 2 presents the results of Pearson and Spearman's correlation analysis between variables. It was shown that abdominal obesity has positive correlation with lipolysis (WC-FFA $\mathrm{r}=0.300 ; \mathrm{p}=0.001$ ), lipid binding protein (WC-FABP4 $r=0.381 ; p=0.000$ ), and insulin resistance (WC-HOMA-IR $r=0.244 ; p=0.005$ ). Angpt13-FGF21 in combination has positive correlation with lipid binding protein (Combination-FABP4 $\mathrm{r}=0.235 ; \mathrm{p}=0.008$ ), inflammation (Combination-TNF $-\alpha \mathrm{r}=0.293 ; \mathrm{p}=0.001$ ) and insulin resistance (Combination-HOMA-IR $\mathrm{r}=0.249$; $\mathrm{p}=0.005$ ) There was a correlation between Angpt13-FFA ( $\mathrm{r}$ $=0,203 ; \mathrm{p}=0,021)$, Angpt13-FABP4 $(\mathrm{r}=0,330 ; \mathrm{p}=0,000)$ and Angpt13-TNF $\alpha(r=0,288 ; p=0,001)$. There was a correlation between FGF21-FABP4 $(r=0,218, p=0,013)$ and FGF21-HOMa-IR ( $r=0,308, p=0,000)$.

Table 3 presents results of regression analysis between each variables.

Table 2. Results of Pearson and Spearman's Correlation Analysis

\begin{tabular}{|c|c|c|c|c|c|c|c|c|}
\hline & & FFA & FABP4 & Angptl3 & FGF21 & TNF- $\alpha$ & HOMA-IR & wc \\
\hline \multirow[t]{2}{*}{ FFA } & $r$ & 1 & $0.502^{\star \star}$ & $0.203^{*}$ & -0.067 & 0.131 & -0.036 & $0.300^{\star \star}$ \\
\hline & $\mathrm{p}$ & - & 0.000 & 0.021 & 0.448 & 0.137 & 0.683 & 0.001 \\
\hline \multirow[t]{2}{*}{ FABP4 } & $r$ & $0.502^{\star \star}$ & 1 & $0.330^{\star \star}$ & $0.218^{\star}$ & 0.022 & $0.226^{\star \star}$ & $0.381^{\star \star}$ \\
\hline & $\mathrm{p}$ & 0.000 & - & 0.000 & 0.013 & 0.805 & 0.010 & 0.000 \\
\hline \multirow[t]{2}{*}{ Angptl3 } & $r$ & $0.203^{\star}$ & $0.330^{\star \star}$ & 1 & $0.203^{\star}$ & $0.288^{\star *}$ & 0.071 & 0.127 \\
\hline & $\mathrm{p}$ & 0.021 & 0.000 & - & 0.020 & 0.001 & 0.425 & 0.148 \\
\hline \multirow[t]{2}{*}{ FGF21 } & $r$ & -0.067 & $0.218^{\star}$ & $0.203^{\star}$ & 1 & $0.204^{\star}$ & $0.308^{\star \star}$ & 0.165 \\
\hline & $p$ & 0.448 & 0.013 & 0.020 & - & 0.020 & 0.000 & 0.061 \\
\hline \multirow[t]{2}{*}{ TNF- $\alpha$} & $r$ & 0.131 & 0.022 & $0.288^{\star \star}$ & $0.204^{\star}$ & 1 & -0.042 & -0.145 \\
\hline & $p$ & 0.137 & 0.805 & 0.001 & 0.020 & - & 0.637 & 0.099 \\
\hline \multirow[t]{2}{*}{ HOMA-IR } & $r$ & -0.036 & $0.226^{\star \star}$ & 0.071 & $0.308^{\star \star}$ & -0.042 & 1 & $0.244^{\star \star}$ \\
\hline & $p$ & 0.683 & 0.010 & 0.425 & 0.000 & 0.637 & - & 0.005 \\
\hline \multirow[t]{2}{*}{ WC } & $r$ & $0.300^{* *}$ & $0.381^{\star \star}$ & 0.127 & 0.165 & -0.145 & $0.244^{\star \star}$ & 1 \\
\hline & $\mathrm{p}$ & 0.001 & 0.000 & 0.148 & 0.061 & 0.099 & 0.005 & - \\
\hline
\end{tabular}

** Correlation significant (level 0,01 (2-tailed))

* Correlation significant (level 0,05 (2-tailed)) 
Table 3. Results of Regression Analysis

\begin{tabular}{lcc}
\hline & R Square & p \\
\hline FFA-WC & 0,107 & 0,000 \\
FFA-FABP4 & 0,252 & 0,000 \\
FFA-Angpt|3 & 0,041 & 0,021 \\
FABP4-WC & 0,145 & 0,000 \\
FABP4-Angptl3 & 0,109 & 0,000 \\
FABP4-FGF21 & 0,047 & 0,013 \\
FABP4-HOMA-IR & 0,061 & 0,005 \\
Angpt|3-FGF21 & 0,041 & 0,020 \\
Angpt|3-TNF- $a$ & 0,049 & 0,011 \\
FGF21-HOMA-IR & 0,046 & 0,014 \\
WC-HOMA-IR & 0,049 & 0,012 \\
Angpt|3-FGF21 Combination-FABP4 & 0,064 & 0,004 \\
Angpt|3-FGF21 Combination-TNF- $\alpha$ & 0,051 & 0,010 \\
Angpt|3-FGF21 Combination-HOMA-IR & 0,057 & 0,007 \\
\hline
\end{tabular}

\section{Discussions}

In this study we found that visceral obesity as shown by increased WC was correlated with FFA and HOMA-IR. Our study confirmed that obesity especially abdominal obesity was related to insulin resistance. Although it is still being debated, it has been found that elevated plasma levels of FFA are a major link between obesity and insulin resistance/T2DM. The study by Boden suggests that plasma levels of FFA are commonly higher in obese people than in non-obese people. The reasons for this include an increased number of fat cells, a decrease in FFA clearance and at a later stage, insulin resistance at the adipose tissue level resulting in increased lipolysis. Increased uptake of FFA into muscle leads to intramyocellular accumulation of fatty acyl-CoA and diacylglycerol (DAG) and activation of protein kinase $\mathrm{C}(\mathrm{PKC})$. It is assumed that activation of PKC interrupts insulin signaling by serine phosphorylation of insulin receptor substrate (IRS)- 1 resulting in a decrease in tyrosine phosphorylation of IRS-1, thereby interrupting insulin signaling and causing insulin resistance (2).
We found that FABP4 had positive correlation with WC, Angpt13, HOMA-IR, FGF21 and Angpt13FGF21 in combination. The lipid chaperone proteins, also known as fatty acid-binding proteins (FABPs), are a group of molecules that coordinate inflammatory and metabolic responses in adipocytes and macrophages. These proteins are a family of $14-$ to $15-\mathrm{kDa}$ proteins that bind with high affinity to hydrophobic ligands such as saturated and unsaturated long-chain fatty acids (10). Obesity is also related to FABP4 and it has been shown that FABP4 is increased in obese adults and is related to metabolic syndrome (11). Expression of FABP4 in adipocytes has been shown to promote lipolysis, increase TNF $-\alpha$ expression, and contribute to insulin resistance (4). We suggest that association between Angpt13 and FABP4 mediates the lipolytic action of Angpt13 on adipocytes. Hormone sensitive lipase (HSL) is responsible for $\approx 50 \%$ of the fatty acids generated by white adipose tissue triacylglycerol hydrolysis and is the rate-limiting enzyme in lipolysis (12). Jun Shen's study showed that HSL interact with FABP4. Deletional mutations of HSL localized the region of HSL that interacts with FABP4 to amino acids $192-200$, and site-directed mutagenesis 
of individual amino acids in this region identified His194 and Glu-199 as critical for mediating the interaction of HSL with FABP4. It appears that FABP4 can increase the hydrolytic activity of HSL through its ability to bind and sequester fatty acids and through its specific proteinprotein interaction, perhaps leading to a conformational change or steric effects on HSL. Jun Shen's observations are consistent with the proposal that FABP4 and HSL constitute a lipolytic complex. This complex functionally results in an increase in the hydrolytic activity of HSL brought about by the physical interaction of HSL with FABP4 and by the ability of FABP4 to bind and sequester fatty acids. Thereby, lipolysis and intracellular trafficking of fatty acids are controlled in an organized fashion (13). We suggest that association between Angpt13 and FABP4 mediates the lipolytic action of Angpt13 on adipocytes, shown by positive correlation between Angpt13-FFA. Shimamura et al. show that there is specific binding of Angpt13 to adipose cells (using fluorescense-labeled protein visually and $125 \mathrm{I}$-labeled protein by the binding analysis) (7). The binding of Angptl3 to adipose cells and association of Angpt13-FABP4 might activate the lipolytic complex between FABP4 and HSL resulting in an increase in the hydrolytic activity of HSL. According to our present study and other previous studies, FABP4 is an independent risk factor for insulin resistance. We suggest that Angpt13 may activate lipolysis in adipose tissue through FABP4 and contribute to insulin resistance risk among obese non diabetic men.

In this study, FGF21 had a significant positive correlation with HOMA-IR. Our study confirmed the finding of Arner et al. which shows that serum FGF21 levels are significantly elevated in overweight/obese subjects and correlated positively with fasting insulin and insulin resistance index (HOMA-IR) (9). FGF21 also has positive correlation with FABP4 and TNF- $\alpha$. Fibroblast growth factor 21 (FGF21) is known as a metabolic regulator with multiple beneficial effects on glucose homeostasis and insulin sensitivity in animal models. Our study has shown that FGF21 is increased in obese non diabetic men, related to increased inflammation and insulin resistance risk. Our study also confirmed the findings of a study by Zhang et al. which show that serum levels of FGF21, which has been suggested as a potential candidate for the treatment of diabetes, are increased in obesity and has demonstrated a strong positive association of serum FGF21 levels with age, several parameters of adiposity (BMI, waist circumference, waist to-hip ratio, and fat percentage), insulin resistance (increased fasting insulin and HOMA-IR and decreased QUICKI), and adverse lipid profiles (increased triglycerides and decreased HDL cholesterol). The paradoxical increase of serum FGF21 in obesity-related disorders might be a defensive response of the human body to counteract the metabolic stress imposed by obesity. Alternatively, obesity may cause resistance to FGF21 actions, leading to its compensatory upregulation (14). We suggested that increased FGF21 concentration occured as a compensation (negative feedback mechanism) to reduce lipolysis and increased sensitivity in non diabetic obese male.

This study showed that Angptl3 had positive correlation with TNF- $\alpha$. We suggest that Angpt13 is related to inflammation and angiogenesis process in obese non diabetic men. A study by Camenisch et al. shows that Angpt13, a liver-specific, secreted factor consisting of an $\mathrm{N}$-terminal coiled-coil domain and the $\mathrm{C}$-terminal fibrinogen (FBN)-like domain. A molecular model of the FBN-like domain of Angpt13 was generated and predicted potential binding to integrins. This hypothesis was experimentally confirmed by the finding that recombinant Angpt 13 bound to $\alpha v \beta 3$ and induced integrin $\alpha v \beta 3$-dependent haptotactic endothelial cell adhesion and migration and stimulated signal transduction pathways characteristic for integrin activation, including phosphorylation of Akt, mitogen- $\alpha$ activated protein kinase, and focal adhesion kinase. When tested in the rat corneal assay, Angpt13 strongly induced angiogenesis with comparable magnitude as observed for vascular endothelial growth factor- $\alpha$. Moreover, the C-terminal FBN-like domain alone was sufficient to induce endothelial cell adhesion and in vivo angiogenesis. Taken together, the data of Camenisch et al. demonstrate that Angpt13 is the first member of the angiopoietin-like family of secreted factors binding to integrin $\alpha v \beta 3$ and suggest a possible role in the regulation of angiogenesis (15). Tumor necrosis factor- $\alpha(\mathrm{TNF}-\alpha)$, one of the major inflammatory cytokines, is known to influence endothelial cell migration. The study by Gao et al. indicates that in response to TNF $-\alpha$, endothelial cells may increase activation and ligation of $\alpha v \beta 3$ while decreasing activation and ligation of $\alpha 5 \beta 1$ integrins to facilitate cell migration, a process essential for vascular wound healing and angiogenesis (16).

We also found positive correlation between Angpt13 and FGF21. We suggest that increasing FGF21 level might occur as a compensatory mechanism to control lipoprotein lipase (LPL) activity in obese non-diabetic men. Angpt13 could increase LPL activity and increase triglyceride concentration in obese mice. Increased FGF21 might down regulate LPL activity, decrease triglyceride concentration and reduce lipotoxicity in obese non diabetic men.

From this study, we concluded that Angptl3 might affect lipolysis and inflammation while FGF21 might affect lipolysis and insulin resistance. The increased FGF21 
concentration might occurred as a compensation (negative feedback mechanism) to reduce lipolysis and increase insulin sensitivity in non diabetic obese males. Further studies might be needed to observe Angpt13 and FGF21 profile in more severe obese population in Indonesia.

\section{Acknowledgements:}

This study was fully funded by Prodia Foundation for Research and Training. We thank the technical staff of Prodia Clinical Laboratory, Clinical Trial and Research and Development Departments for their valuable assistance in undertaking specimen collection in the field and for the biochemical assessments.

\section{Conflict of Interest:}

There is no conflict of interest related to this study

\section{References:}

1. Zhang C, Rexrade KM, van Dam RM, Li TY, Hu FB. Abdominal Obesity and the Risk of All-Cause, Cardiovascular and Cancer Mortality: Sixteen Years of Follow-Up in US Women. Circulation 2008; 117 : 1658-1667

2. Boden G. Free Fatty Acid as Target For Therapy. Curr Opin Endocrinol Diabetes 2004; 11 : 258-263

3. Nguyen MTA, Satoh H, Favelyukis S, Babendure JL, Imamura T, Sbodio JI, et al. JNK and Tumor Necrosis Factor-a Mediate Free Fatty Acid-induced Insulin Resistance in 3T3-L1 Adipocytes. J Biol Chem 2005; 280 : 3536135371

4. Boord JB, Fazio S, Linton MF. Cytoplasmic Fatty AcidBinding Protein: Emerging Roles in Metabolism and Atherosclerosis. Curr Opin Lipidol 2002; 13 : 141-147

5. Wallace TM, Levy JC, Matthews DR. Use and Abuse of HOMAIR Modeling. Diab Care 2004; 27 : 1487-1495
6. Oike Y, Akao M, Kubota Y, Suda T. Angiopoietin-Like Proteins Potential New Targets for Metabolic Syndrome Therapy. Trends Mol Med 2005; 11 : 473-479

7. Shimamura M, Matsuda M, Kobayashi S, Ando Y, Ono M, Koishi $\mathrm{R}$, et al. Angiopoietin-like Protein 3, a Hepatic Secretory Factor, Activates Lipolysis in Adipocyte. Biochem Biophys Res Commun 2003; 301 : 604-609

8. Moore DD. Sister Act. Science 2007; 316 : 1436-1438

9. Arner P, Pettersson A, Mitchell PJ, Dunbar JD, Kharitonenkov A, Ryden M. FGF21 Attenuates Lipolysis in Human Adipocytes-APossible Link to Improved Insulin Sensitivity. FEBS Letters 2008; 582: 1725-1730

10. Furuhashi M, Fucho R, Gorgun CZ, Tuncman G, Cao H, Hotamisligil GS. Adipocyte/Macrophage Fatty AcidBinding Proteins Contribute to Metabolic Deterioration Through Actions in Both Macrophages and Adipocytes in Mice. J Clin Invest 2008; 118 : 2640-2650

11. Reinehr T, Stoffel-Wagner B, Roth CL. Adipocyte Fatty AcidBinding Protein in Obese Children Before and After Weight Loss. Metab Clin Exp 2007; 56 : 1735-1741

12. Jenkins-Kruchten $A E$, Bennaars-Eiden $A$, Ross JR, Shen WJ, Kraemer FB, Bernlohr DA. Fatty- $\alpha$ cid Binding ProteinHormone Sensitive Lipase Interaction. J Biol Chem 2003; 278 : 47636-47643

13. Jun Shen W, Liang Y, Hong R, Patel S, Natu V, Sridhar K, et al. Characterization of the Functional Interaction of Adipocyte Lipid-binding Protein with Hormone-Sensitive Lipase. J Biol Chem 2001; 276 : 49443-49448

14. Zhang X, Yeung DCY, Karpisek M, Stejskal D, Zhou SG, Liu F, et al. Serum FGF21 Levels are Increased in Obesity and Independently Associated with the Metabolic Syndrome in Humans. Diabetes 2008; 57 : 1246-1253

15. Camenisch G, Pisabarro MT, Sherman D, Kowalski J, Nagel M, Hass P, et al. Angptl3 Stimulates Endothelial Cell Adhesion and Migration via Integrin $\alpha v \beta 3$ and Induces Blood Vessel Formation in vivo. J Biol Chem 2002; 277 : 17281-17290

16. Gao B, Saba TM, Tsan MF. Role of Alpha Beta 3-Integrin in TNF- $\alpha$ Ipha-Induced Endothelial Cell Migration. Am J Physiol Cell Physiol 2002; 283 : 1196-1205 